\title{
SPONTANEOUS CURRENT GENERATION IN COSMIC STRINGS (DAMTP-93/34)
}

\author{
Patrick PETER \\ Department of Applied Mathematics and Theoretical Physics, \\ University of Cambridge, Silver Street, Cambridge CB3 9EW (UK), \\ and \\ Département d'Astrophysique Relativiste et de Cosmologie \\ Observatoire de Paris-Meudon, UPR 176 CNRS, 92195 MEUDON Cedex (France)
}

(September 20, 2021)

\begin{abstract}
It is shown that in models including the standard electroweak theory and for some particular values of the underlying parameters, electric currents can be spontaneously generated in cosmic strings, without the need of any external field (e.g., electric or magnetic) as is required in most models. This mechanism is then shown to break spontaneously the Lorentz invariance along the initially Goto-Nambu string. The characteristic time needed for the current to build up is estimated and found to lowest order to depend only on the mass of the intermediate $W$ vector boson and the fine structure constant.
\end{abstract}

PACS Numbers: 98.80.Cq, 11.17.+y, 12.15.Cc

\section{INTRODUCTION}

Cosmic strings [1] are linear vortex defects predicted to be formed at a cosmological phase transition during which the vacuum manifold would not be simply connected. The first interest in studying them comes from the fact that since a typical grand unified theory (GUT) predicts a few phase transitions (whose order, as far as the strings are concerned, does actually not matter [2]), and because the vacuum structure needed to form strings happens to be generically realized, one can, following Vilenkin [3], reasonnably assume that cosmic strings have an existence probability of at least $\frac{1}{2}$. Although they are not the only possible topological defects that could be formed in such phase transitions, they have the advantage, as compared for instance with domain walls and monopoles which must be somehow inflated away [1.4], to be at present compatible (as are as well the textures [5]) with all existent cosmological data [6], while being also possibly responsible for the formation of large scale structure [7] and the observed anisotropies in the cosmic microwave background (CMBR) [8]. Most models based on these strings assume that they are generated at the GUT phase transition, so that the dimensionless parameter $G U$, with $G$ the Newton constant and $U$ the energy per unit string length, giving the expected relative order of magnitude of any gravitational effect due to these strings (e.g., light deflection [9] or CMBR temperature fluctuations [6]), was assumed to be $\sim 10^{-6}$.

Another kind of strings was proposed by Witten [10] in 1985 who pointed out the possibility that bosonic or fermionic superconducting currents could be trapped in the strings, thereby inducing many electromagnetic effects, such as, for instance, a new scenario for structure formation [11]. Shortly thereafter, it was shown by Davis and Shellard [12] and independently by Carter [13] that, although the regular strings cannot be potentially responsible for a cosmological catastrophe (i.e., the remnant mass density would not exceed the critical density) because of gravitational radiation and the absence of any stabilising mechanism, the situation was completely different for current-carrying string loops since in the latter case, there exists centrifugally supported equilibrium configurations (called vortons [12] or rings [13]) which would overfill the Universe [14] by many orders of magnitude if they were stable (a point which still demands further clarification and is presently under investigation 15]), this stabilizing mechanism being enhanced when electromagnetic corrections are taken into account [16], and should not be confused with the much less efficient "spring", or magnetostatic support mechanism [11,17, 18].

The Witten mechanism to produce currents in cosmic strings has been studied by many authors, interested in particular by their internal microscopic structure [17], and who exhibited clearly the characteristic features of what should be expected in these objects, such as the existence of a maximum (spacelike) current (or the current quenching phenomenon) and a phase frequency threshold (for timelike currents). Independently, a "macroscopic" formalism 19] was derived that allows one in principle to evaluate the dynamics of any current-carrying string configuration once its equation of state, relating the energy per unit length $U$ to the tension $T$, is given. This equation of state, for the Witten simple model describing strings, and whose properties are believed to be qualitatively (if not quantitatively) similar to more complicated (and realistic) strings models, was indeed obtained (albeit unfortunately only numerically), so that it has now become possible to study realistically the cosmological importance of superconducting cosmic strings, 
and their astrophysical, gravitationally induced, signature, since the structure of the spacetime surrounding a string of any kind is also known [20].

The purpose of this article is to show that there exists yet another mechanism by which a string can become not only superconducting, but also current-carrying without invoking any extra external field (e.g., electromagnetic), hence the name spontaneous current generation for this mechanism, comparable in many respects to a similar mechanism existing in ${ }^{3} \mathrm{He}$ vortex lines [21]. This phenomenon occurs for particular values of the underlying microscopic parameters, when the current-carrier field is neither a scalar nor a fermionic field (these cases being essentially equivalent due to the two dimensional description of the vortex), but a charged-coupled vector field such as the intermediate $W^{ \pm}$in the electroweak theory. To illustrate this mechanism in the most realistic possible way, we shall consider a simple stringforming extension 222 of the standard electroweak theory [23] (this latter model being string-free since its vacuum manifold, isomorphic to the 3 -sphere $S^{3}$, is simply connected). Other motivations [24] such as supersymmetry or superstrings inspired models also lead, generally as a low energy limit, to the model we wish to consider, namely that in which an extra- $U(1)$ is gauged, this new symmetry being spontaneously broken. It is interesting to notice that because of the large number of experimentally testable phenomenological consequences of such a model, the energy scale of the symmetry breaking involved $E_{Z^{\prime}}$ say, and thus the energy per unit length of the corresponding strings, is in fact constrained to exceed 25] $300-500 \mathrm{GeV}$ (depending on the couplings), which is actually close to the upper limit provided by the vorton mechanism [12,14], namely $E_{Z^{\prime}} \lesssim 10 \mathrm{TeV}$.

We shall first introduce our string forming model, as well as the vortex solutions themselves, in the simple case to begin with where no current is flowing in the strings, i.e., the so-called Nielsen-Olesen [26] solutions, or Kibble type vortices. Then we go on the spontaneous current generation itself which is shown to be be due to an electromagnetic instability of the vacuum for massless $W$ field at zero temperature, and finally discuss how the phenomenon is responsible for a spontaneous breaking of the Lorentz boost symmetry along the initially Kibble-like string.

\section{KIBBLE VORTICES BEYOND THE ELECTROWEAK MODEL.}

The electroweak theory [23] is based on the spontaneous breaking of the $S U(2)_{L} \times U(1)_{Y}$ symmetry by means of an $S U(2)$ doublet Higgs field $H$ down to the electromagnetic $U(1)$ symmetry. This means that the vacuum has the topology of the quotient group $S U(2) \times U(1) / U(1)$, which is isomorphic to $S U(2)$, i.e., it has the topology of the 3 -sphere and is therefore simply connected. As a result, topologically stable cosmic strings are not present in this model (and in fact, due to the experimental bound on the Higgs mass $M_{H} \gtrsim 65 \mathrm{GeV}$ [25], even string-like solutions in this model are dynamically unstable [27,28]). In order to investigate the structure of cosmic strings in a realistic model that would take the electroweak theory into account, it is thus necessary to modify this theory first. There are basically two different approaches that can be followed to extend this model. The first one consists in assuming the Higgs realisation of the symmetry breaking not to be fundamental, and to consider instead dynamical symmetry breaking such as in the chiral approach [29] involving the $S U(2)_{L} \times S U(2)_{R}$ symmetry. This leads to the existence of semi-topological defects [because only one direction of $S U(2)_{R}$ is actually gauged], which may be shown [30] to be dynamically stable and moreover superconducting. The second approach, the one we shall follow, is to regard the Higgs representation, and thus the Higgs field $H$ itself, as fundamental, and to extend the gauge group. It turns out that the most simple such extension one can think of, consisting in an extra $U(1)$, also generates topologically stable (and superconducting) cosmic strings [22].

The string-forming model we shall now examine is the following (this section is essentially useful to fix the notation used throughout): initially, the symmetry $S U(2)_{L} \times U(1)_{Y} \times U(1)_{F}$ (with $F$ the extra hypercharge) is broken down to $S U(2)_{L} \times U(1)_{Y}$ by means of a Higgs field $\Phi$, and this is followed by the usual electroweak phase transition. The model is minimal in the sense that we assign a vanishing $F$ hypercharge for the $H$ field, and symmetrically assume $\Phi$ to be an $S U(2)$ singlet. We shall altogether neglect the fermionic sector of the model, but it may be remarked that the hypercharge $F$, with the previous assignement made on the Higgs fields, coincides with $B-L$ (up to a normalisation factor absorbable in the fermionic fields) in this sector, and that even though $U(1)_{F}$ is broken, the baryonic and leptonic numbers $B$ and $L$ are conserved. Also the model will be anomaly-free provided one includes a right-handed neutrino.

We therefore start with the Lagrangian density (again, without the fermions)

$$
\mathcal{L}=-\frac{1}{4} \vec{F}_{\mu \nu} \cdot \vec{F}^{\mu \nu}-\frac{1}{4} G_{\mu \nu} G^{\mu \nu}-\frac{1}{4} H_{\mu \nu} H^{\mu \nu}-\left(D_{\mu} H\right)^{\dagger} D^{\mu} H-\left(D_{\mu} \Phi\right)^{\star} D^{\mu} \Phi-V(H, \Phi),
$$

where the (classical) potential between the Higgs fields is (we assume that both phase transitions are second order so we neglect the logarithmic corrections [2]31] in this zero temperature effective theory, see, however, Ref. [32] on that point) 


$$
V(H, \Phi)=\lambda_{H}\left(H^{\dagger} H-\frac{v_{H}^{2}}{2}\right)^{2}+\lambda_{\phi}\left(|\Phi|^{2}-\frac{v_{\phi}^{2}}{2}\right)^{2}+f\left(H^{\dagger} H-\frac{v_{H}^{2}}{2}\right)\left(|\Phi|^{2}-\frac{v_{\phi}^{2}}{2}\right),
$$

and we have set the covariant derivative

$$
D_{\mu} \equiv \partial_{\mu}-i g \frac{\vec{T}}{2} \cdot \vec{A}_{\mu}-i g^{\prime} \frac{Y}{2} B_{\mu}-i q \frac{F}{2} C_{\mu}
$$

with $T^{i}$ the generators of $S U(2)_{L}$ in the representation of the particle upon which the derivative acts, $g, g^{\prime}$ and $q$ the gauge coupling constants of $S U(2)_{L}, U(1)_{Y}$ and $U(1)_{F}$ respectively, and the kinetic terms of the gauge vectors are expressed through

$$
\begin{gathered}
F_{\mu \nu}^{i}=\partial_{\mu} A_{\nu}^{i}-\partial_{\nu} A_{\mu}^{i}+g \varepsilon^{i j k} A_{\mu}^{j} A_{\nu}^{k}, \\
G_{\mu \nu}=\partial_{\mu} B_{\nu}-\partial_{\nu} B_{\mu} \quad, \quad H_{\mu \nu}=\partial_{\mu} C_{\nu}-\partial_{\nu} C_{\mu} .
\end{gathered}
$$

The Higgs doublet is understood as $H=\left(H^{+}, H^{0}\right)$, and its vacuum expectation value (VEV) is experimentally known to be $\sqrt{\left\langle|H|^{2}\right\rangle_{0}}=v_{H} / \sqrt{2} \simeq 174 \mathrm{GeV}$.

We are now interested in a vortex solution of this model, of the kind proposed by Nielsen and Olesen [26], for the $\Phi$ field. Since we are concerned by classical solutions, it is necessary that we fix the gauges. For the string-forming fields, there is a particularly convenient gauge choice: if the vortex solution is taken to be aligned along the $z$ axis (which is always possible since the curvature effects can be locally neglected), we can choose a cylindrical coordinate system, and in this system, the phase of the $\Phi$ field is identified with the angular coordinate $\theta$. The Nielsen-Olesen vortex solution then takes the simple form

$$
\Phi=\varphi(r) \exp i n \theta
$$

with $n$ the winding number 1.,2.4.

Let us now turn to the electroweak fields. Because of the disjoint structure of the initial invariance, we have not lost any freedom in going to the vortex gauge. We can thus choose the most convenient gauge with regard to the subsequent interpretation, namely the unitary gauge, in which only the neutral component of $H$ is considered: $H=[0, h(r) / \sqrt{2}]$. Before going any further in the resolution of the Euler-Lagrange equations for this system, we wish to examine in more details what occurs in the strings core.

The string solution is defined as the set of points in space where $\Phi=0$. Moreover, the vacuum (or the false vacuum in the case of the strings core) should represent a minimum of the potential (2). Varying this potential for $h$ and $\varphi$, we see that the extremization yields two differents possibilities, namely, far from the strings core, i.e., in the usual vacuum,

$$
h=v_{h} \text { and } \varphi=v_{\phi} / \sqrt{2},
$$

whereas in the strings core with $\varphi=0$, then $h$ should satisfy (not taking the kinetic terms into account for the moment)

$$
h^{2}=v_{H}^{2}+f \frac{v_{\phi}^{2}}{\lambda_{H}}
$$

from which we can conclude that two cases may occur in principle. The first case, already studied elsewhere [22], is for $f>f_{\text {crit }}$, with

$$
f_{\text {crit }}=-\lambda_{H}\left(\frac{v_{H}}{v_{\phi}}\right)^{2}
$$

which corresponds to a shift in the $S U(2)$ doublet Higgs VEV at $r=0$. The second case, to which we now turn definitely, is for $f \leq f_{\text {crit }}$. If the underlying parameters are such that this inequality is satisfied, then there is no real solution to Eq. (8). Thus, one finds that the real minimum of the potential is now at $h=0$ as long as $\varphi \leq \varphi_{\min }$, where $\varphi_{\min }^{2}=v_{\phi}^{2} / 2-\lambda_{H} v_{H}^{2} /|f|$ [given by inserting a nonzero value for $\sigma$ into Eq. (8) and solving for $h=0$ ].

Fig. 1 illustrates the internal string structure which is obtained when the kinetic terms are included. This figure represents a solution of the field equations derived from the Lagrangian (1) under the gauge assumptions and with the vortex solution (6), with zero vector fields $A_{i \mu}$ and $B_{\mu}$. This solution was obtained by means of a successive over 
relaxation method [3], and the distances are in units of the inverse $\Phi$ mass $\left(\lambda_{\phi} v_{\phi}\right)^{-1}$. More details concerning the numerical procedure itself and the stability of the solution can be found in Ref. [22], but here, and in particular in the next section, we shall be mainly interested in what occurs close to the strings core, namely the symmetry restoration. For the time being, let us just remark that since the Higgs field $h$ is real, there is no associated current with it, so the fact that it be trapped in the string, its VEV varying from $r=0$ to $r \rightarrow \infty$, merely changes the actual value of the string's energy per unit length, but otherwise does not break the Lorentz boost invariance along the string. Therefore, setting the stress-energy tensor in the form

$$
T^{\mu \nu}=U u^{\mu} u^{\nu}-T v^{\mu} v^{\nu},
$$

with $u$ and $v$ two unit timelike and spacelike vector respectively, tangent to the strings worldsheet, $U$ being the energy per unit length and $T$ the tension, the Lorentz invariance requires, whether there is a Higgs condensate or not, that the equation of state be that of Goto-Nambu, i.e., $U=T=$ Cte [1. 19]. This is important because once the current-generation mechanism which we will investigate in the next section has been at work, this degeneracy in the stress-energy tensor eigenvalues is spontaneously raised, so the Lorentz invariance is spoiled.

\section{INTERMEDIATE BOSON INSTABILITY.}

The electroweak vacuum surrounding a cosmic string of the kind we just investigated is in fact not stable. This can be seen as follow: in the standard vacuum, the $W^{ \pm}$particles are charged and massive because of the Higgs field $H$ VEV. Now, close to the string core, as we have just seen, this VEV actually vanishes so the $W^{ \pm}$particles become charged and massless. As a result, they can be created by pair through any fluctuation of the electromagnetic field, but since they are charged, they can actually be considered themselves as the sources for these electromagnetic fluctuations. More precisely, as will be shown in this section, fluctuations in the $W$ field yield a corresponding nonvanishing $A$ and $Z$, with nonzero gradients. This implies nonzero electric and magnetic fields which are used as negative masses for the $W$ particles. The vacuum surrounding a cosmic string is thus unstable and there is a spontaneous current generation in the form of $W$ flowing along the strings.

To see how this phenomenon actually occurs, let us concentrate on the stress energy tensor $T^{\mu \nu}$ given by

$$
T^{\mu \nu}=-2 g^{\mu \alpha} g^{\nu \sigma} \frac{\delta \mathcal{L}}{\delta g^{\alpha \sigma}}+g^{\mu \nu} \mathcal{L}
$$

and in particular the energy density $\mathcal{U}=T^{t t}$. Setting as usual [17] $Q(r)=n-\frac{1}{2} q C_{\theta}, W_{\mu}^{ \pm}=\left(A_{1 \mu} \mp i A_{2 \mu}\right) / \sqrt{2}$, $Z_{\mu}=c A_{3 \mu}-s B_{\mu}, A_{\mu}=s A_{3 \mu}+c B_{\mu}, s \equiv \sin \theta_{W}, c \equiv \cos \theta_{W}, \tan \theta_{W} \equiv g^{\prime} / g$, and using the vortex ansatz (6) gives, if one considers only a configuration where radial electric and orthoradial magnetic fields are present (i.e., with $A_{z}(r)$, $A_{t}(r), Z_{z}(r)$ and $Z_{t}(r)$ the only nonvanishing components of the photonic and the $Z$ fields)

$$
\begin{aligned}
\mathcal{U} & =\frac{1}{2} h^{\prime 2}+\varphi^{\prime 2}+\frac{\varphi^{2} Q^{2}}{r^{2}}+\frac{2 Q^{\prime 2}}{r^{2} q^{2}}+\frac{\lambda_{H}}{4}\left(h^{2}-v_{H}^{2}\right)^{2}+\lambda_{\phi}\left(\varphi^{2}-\frac{v_{\phi}^{2}}{2}\right)^{2}+\frac{1}{2} f\left(h^{2}-v_{H}^{2}\right)\left(\varphi^{2}-\frac{v_{\phi}^{2}}{2}\right) \\
& +2 W_{\mu t}^{+} W_{t}^{-\mu}+\frac{1}{2} W_{\mu \nu}^{+} W^{-\mu \nu}+\frac{1}{2}\left(A_{z}^{\prime 2}+A_{t}^{\prime 2}+Z_{z}^{\prime 2}+Z_{t}^{\prime 2}\right)+\frac{g^{2}}{8 c^{2}} h^{2}\left(Z_{z}^{2}+Z_{t}^{2}\right) \\
& +2 i g\left\{W_{\mu t}^{+}\left[W_{t}^{-}\left(s A^{\mu}+c Z^{\mu}\right)-W^{-\mu}\left(s A_{t}+c Z_{t}\right)\right]+W_{\mu t}^{-}\left[W^{+\mu}\left(s A_{t}+c Z_{t}\right)-W_{t}^{+}\left(s A^{\mu}+c Z^{\mu}\right)\right]\right\} \\
& +\frac{1}{2} i g\left\{W_{\mu \nu}^{+}\left[W^{-\nu}\left(s A^{\mu}+c Z^{\mu}\right)-W^{-\mu}\left(s A^{\nu}+c Z^{\nu}\right)\right]+W_{\mu \nu}^{-}\left[W^{+\mu}\left(s A^{\nu}+c Z^{\nu}\right)-W^{+\mu}\left(s A^{\nu}+c Z^{\nu}\right)\right]\right\} \\
& +\frac{1}{4} g^{2} h^{2}\left(\left|W_{r}\right|^{2}+\left|W_{z}\right|^{2}+\frac{1}{r^{2}}\left|W_{\theta}\right|^{2}+\left|W_{t}\right|^{2}\right) \\
& +i g\left[\left(s A_{z}^{\prime}+c Z_{z}^{\prime}\right)\left(W_{r}^{-} W_{z}^{+}-W_{z}^{-} W_{r}^{+}\right)+\left(s A_{t}^{\prime}+c Z_{t}^{\prime}\right)\left(W_{r}^{-} W_{t}^{+}-W_{t}^{-} W_{r}^{+}\right)\right] \\
& -g^{2}\left\{\left|\left(s A_{z}+c Z_{z}\right) W_{z}^{+}+\left(s A_{t}+c Z_{t}\right) W_{t}^{+}\right|^{2}\right. \\
& +\frac{1}{2}\left(W_{r}^{+} W_{t}^{-}-W_{r}^{-} W_{t}^{+}\right)^{2}+\frac{1}{2}\left(W_{z}^{+} W_{t}^{-}-W_{z}^{-} W_{t}^{+}\right)^{2}+\frac{1}{2}\left(W_{r}^{+} W_{z}^{-}-W_{r}^{-} W_{z}^{+}\right)^{2} \\
& +\frac{1}{2 r^{2}}\left[\left(W_{r}^{+} W_{\theta}^{-}-W_{r}^{-} W_{\theta}^{+}\right)^{2}+\left(W_{z}^{+} W_{\theta}^{-}-W_{z}^{-} W_{\theta}^{+}\right)^{2}+\left(W_{t}^{+} W_{\theta}^{-}-W_{t}^{-} W_{\theta}^{+}\right)^{2}\right] \\
& \left.-\left(\left|W_{r}\right|^{2}+\left|W_{z}\right|^{2}+\frac{1}{r^{2}}\left|W_{\theta}\right|^{2}+\left|W_{t}\right|^{2}\right)\left[\left(s A_{z}+c Z_{z}\right)^{2}+\left(s A_{t}+c Z_{t}\right)^{2}\right]\right\}
\end{aligned}
$$


where a prime means differentiation with respect to the radial coordinate $r$. Let us now consider the quadratic terms in $W^{ \pm}$that are present in Eq. (12), i.e., the effective mass matrix $M_{i j}^{2}$, defined by

$$
\left.M_{i j}^{2} \equiv 2 \frac{\delta \mathcal{U}}{\delta W_{i}^{+} \delta W_{j}^{-}}\right|_{W_{i}^{\mu}=0=A^{\mu}=Z^{\mu}} .
$$

Because of the coupling between the $W$ fields and the gradients of the photon and the $Z$ fields, this mass matrix is in fact nondiagonal, and one can easily derive the eigenvalues as

$$
\begin{gathered}
m_{1}^{2}=\frac{1}{2} g^{2} h^{2}, \\
m_{2}^{2}=\frac{1}{2} g^{2} h^{2}+2 g \sqrt{\left(s A_{z}^{\prime}+c Z_{z}^{\prime}\right)^{2}+\left(s A_{t}^{\prime}+c Z^{\prime}+t\right)^{2}}, \\
m_{3}^{2}=\frac{1}{2} g^{2} h^{2}-2 g \sqrt{\left(s A_{z}^{\prime}+c Z_{z}^{\prime}\right)^{2}+\left(s A_{t}^{\prime}+c Z^{\prime}+t\right)^{2}},
\end{gathered}
$$

where, to first order in $g$, the eigenvectors are

$$
\begin{gathered}
W_{1}^{-}=\frac{1}{\sqrt{\beta_{z}^{2}+\beta_{t}^{2}}}\left(\beta_{t} W_{z}^{-}-\beta_{z} W_{t}^{-}\right), \\
W_{2}^{-}=\frac{1}{\sqrt{2}}\left[W_{r}^{-}-\frac{i}{\sqrt{\beta_{z}^{2}+\beta_{t}^{2}}}\left(\beta_{z} W_{z}^{-}+\beta_{t} W_{t}^{-}\right)\right], \\
W_{3}^{-}=\frac{1}{\sqrt{2}}\left[W_{r}^{-}+\frac{i}{\sqrt{\beta_{z}^{2}+\beta_{t}^{2}}}\left(\beta_{z} W_{z}^{-}+\beta_{t} W_{t}^{-}\right)\right],
\end{gathered}
$$

and we have set $\beta_{a}=g\left(s A_{a}^{\prime}+c Z_{a}^{\prime}\right), a=z, t$ the radial (respectively orthoradial) component of the electric (resp. magnetic) field.

It can be remarked on Eqs. (14), (15) and (16) that $W_{3}$ has a nonpositive definite mass, which is not the case for all other components of this gauge field. Therefore, since we are seeking a minimum energy configuration, it seems safe to assume $W_{1}=W_{2}=0=W_{\theta}$. Setting, for simplicity, $W_{3}^{+} \equiv W$, one has

$$
\begin{gathered}
W_{r}^{+}=\frac{1}{\sqrt{2}} W, \\
W_{z}^{+}=\frac{i \beta_{z}}{\sqrt{2\left(\beta_{z}^{2}+\beta_{t}^{2}\right)}} W, \\
W_{t}^{+}=\frac{i \beta_{t}}{\sqrt{2\left(\beta_{z}^{2}+\beta_{t}^{2}\right)}} W,
\end{gathered}
$$

where now $\beta_{z}$ and $\beta_{t}$ are arbitrary. We recover the previous results [22] $W_{r}^{-}= \pm i W_{z}^{-}$or $W_{r}^{-}= \pm i W_{t}^{-}$for the purely magnetic or electric cases respectively (i.e., when only one component of $A$ and $Z$ is explicitely considered).

Under the assumptions given by Eqs. (20), (21) and (22), it is now simple to derive the effective potential for the $W$ field, namely:

$$
V(W)=\frac{1}{2} m_{3}^{2}|W|^{2}+g^{2}|W|^{4},
$$

so this field effectively behaves as a Higgs "scalar" in the region of parameter space where its squared mass is negative. This is indeed possible close to the strings core since there, as we have seen, still in the case where $f<f_{\text {crit }}$, the Higgs field $h$ vanishes, so to first order in $g$, Eq. (16) implies that $m_{3}^{2}$ is actually negative. Thus, any fluctuation in $W$, the latter being coupled with the photon, will generate a small fluctuation in $A_{z}, A_{t}, Z_{z}$ and $Z_{t}$, which, if the initial perturbation was axisymmetric, will produce an electric or a magnetic field. Since $W$ is effectively massless in the core of the string, the energy in the electromagnetic perturbation is already sufficient to create a pair $W^{+} W^{-}$, which can in turn be seen as the source for the electromagnetic fields. Since these electromagnetic fields are necessary to support the $W$ condensate in the strings core, one is led to the conclusion that a current has been spontaneously generated. We shall now investigate in more details this mechanism. 


\section{SPONTANEOUS CURRENT GENERATION.}

To exhibit the instability of the electroweak vacuum surrounding a Nielsen-Olesen string (6), we turn to the EulerLagrange equations derivable from the Lagrangian (11), which we expand to first order in the coupling constant $g$ and to lowest order in the various fields involved to consider the case of a perturbation in $W_{3}$ [given by Eq. (19)]. For the photon, we have

$$
\partial_{\beta}\left\{r\left[A^{\alpha \beta}+i g s\left(W^{-\alpha} W^{+\beta}-W^{-\beta} W^{+\alpha}\right)\right]\right\}=\operatorname{rigs}\left(W^{-\alpha \beta} W_{\beta}^{+}-W^{+\alpha \beta} W_{\beta}^{-}\right),
$$

a similar equation applying for the $Z$ field with $\sin \theta_{W}$ replaced by $\cos \theta_{W}$, so also similar conclusions can actually be drawn for both fields, and

$$
\partial_{\beta}\left\{r\left[W^{-\alpha \beta}-i g W^{-\alpha}\left(s A^{\beta}+c Z^{\beta}\right)+i g W^{-\beta}\left(s A^{\alpha}+c Z^{\alpha}\right)\right]\right\}=\operatorname{rigs}\left[W^{-\beta \alpha}\left(s A_{\beta}+c Z_{\beta}\right)+\left(s A^{\beta \alpha}+c Z^{\beta \alpha}\right) W_{\beta}^{-}\right],
$$

close to the strings core, in the symmetry restoration region where $h=0$. In fact, because there, $h=0$, neglecting a possible backreaction due to outer region couplings, we see that the background Nielsen-Olesen string is essentially unaffected by the inclusion of the $W, A$ and $Z$ fields.

We shall now examine a perturbation in $W_{3}=W$ in the form $W=|W(r)| e^{i \omega t}$. Inserting this form into Eq. (24) yields

$$
\begin{gathered}
\frac{\partial^{2} A_{z}}{\partial z \partial r}-\frac{\partial^{2} A_{t}}{\partial t \partial r}-\frac{\partial^{2} A_{r}}{\partial z^{2}}+\frac{\partial^{2} A_{r}}{\partial t^{2}}=0 \\
\frac{\partial^{2} A_{r}}{\partial z \partial r}-\frac{\partial^{2} A_{z}}{\partial r^{2}}-\frac{\partial^{2} A_{t}}{\partial z \partial t}+\frac{\partial^{2} A_{z}}{\partial t^{2}}+\frac{1}{r} \frac{\partial A_{r}}{\partial z}-\frac{1}{r} \frac{\partial A_{z}}{\partial r}=\frac{g s \beta_{z}}{\sqrt{\beta_{z}^{2}+\beta_{t}^{2}}}\left[\left(\frac{\beta_{t} \omega}{\sqrt{\beta_{z}^{2}+\beta_{t}^{2}}}-\frac{1}{r}\right)|W|^{2}-3|W| \frac{d|W|}{d r}\right], \\
\frac{\partial^{2} A_{r}}{\partial r \partial t}-\frac{\partial^{2} A_{t}}{\partial r^{2}}+\frac{\partial^{2} A_{z}}{\partial z \partial t}-\frac{\partial^{2} A_{t}}{\partial z^{2}}+\frac{1}{r} \frac{\partial A_{r}}{\partial t}-\frac{1}{r} \frac{\partial A_{t}}{\partial r}=\frac{g s \beta_{t}}{\sqrt{\beta_{z}^{2}+\beta_{t}^{2}}}\left[\left(\frac{\beta_{t} \omega}{\sqrt{\beta_{z}^{2}+\beta_{t}^{2}}}-\frac{1}{r}\right)|W|^{2}-3|W| \frac{d|W|}{d r}\right]
\end{gathered}
$$

equations in which we shall assume for now on that $\partial_{z}$ is ignorable, and we will denote the differentiation with respect to the radial coordinate $r$ by a prime, whereas a dot will mean a derivative with respect to time. We shall also work in the Lorentz gauge $\nabla_{\mu} A^{\mu}=0$ since in this gauge the equations for the various components of $A$ are decoupled. Actually, the gauge condition together with the field equation (26) with $\partial_{z}$ ignorable yields, upon differentiation with respect to $r$

$$
\dot{A}_{r}^{\prime}+\frac{1}{r} \dot{A}_{r}=\ddot{A}_{t}
$$

and inserting this into Eq. (26) yields

$$
\ddot{A}_{r}=A_{r}^{\prime \prime}+\frac{1}{r} A_{r}^{\prime}-\frac{1}{r^{2}} A_{r}
$$

so that setting $A_{r}=f(t) g(r)$ gives

$$
\frac{\ddot{f}}{f}=\frac{\Delta_{2} g}{g}-\frac{1}{r^{2}}=-\alpha^{2},
$$

with $\alpha$ a constant and $\Delta_{2}$ the two dimensionnal Laplacian in the transverse plane. So $f \propto e^{i \alpha t}$, and the radial dependent part obeys a Schrödinger equation

$$
-\Delta_{2} g+\frac{1}{r^{2}} g=\alpha^{2} g,
$$

with a positive definite potential $r^{-2}$. Therefore, the eigenvalues $\alpha^{2}$ of Eq. (32) are all positive and the string state is stable against $A_{r}$ perturbations. Thus, neglecting $A_{r}$ in the resulting stationnary configurations is justified and we shall consistently set $A_{r}=0$ in what follows. 
Inserting Eq. (29) into Eq. (28) is the last step toward the definite equations describing the dynamics of $A_{z}$ and $A_{t}$ when a perturbation in $W_{3}$ is applied, and we find

$$
\begin{aligned}
& \ddot{A}_{z}-A_{z}^{\prime \prime}-\frac{1}{r} A_{z}^{\prime}=\frac{g s \beta_{z}}{\sqrt{\beta_{z}^{2}+\beta_{t}^{2}}}\left[\left(\frac{\beta_{t} \omega}{\sqrt{\beta_{z}^{2}+\beta_{t}^{2}}}-\frac{1}{r}\right)|W|^{2}-3|W| \frac{d|W|}{d r}\right], \\
& \ddot{A}_{t}-A_{t}^{\prime \prime}-\frac{1}{r} A_{t}^{\prime}=\frac{g s \beta_{t}}{\sqrt{\beta_{z}^{2}+\beta_{t}^{2}}}\left[\left(\frac{\beta_{t} \omega}{\sqrt{\beta_{z}^{2}+\beta_{t}^{2}}}-\frac{1}{r}\right)|W|^{2}-3|W| \frac{d|W|}{d r}\right],
\end{aligned}
$$

out of which the spontaneous current mechanism can be clearly exhibited.

The linearized field equations (33) and (34) have a first immediate consequence, namely that $\beta_{t} A_{z}=\beta_{z} A_{t}$. It turns out that this relation is still valid when the whole set of classical field equations are used, so the overall field configuration is in fact determined by the value of the ratio $\beta_{z} / \beta_{t}$. We will return to that point later. Moreover, Eqs. (33) and (34), being inhomogeneous because of the source term due to the $W$ field [a direct consequence of the nonabelian nature of $S U(2) \times U(1)]$, the configuration $\left(A_{z}=0\right.$ and $\left.A_{t}=0\right)$ is not solution of the field equations, and more generally there is also no solution with vanishing gradient. But this is precisely the condition for the squared mass $m_{3}^{2}$ to be nonpositive definite. Thus, we know that there exist unstable modes in Eq. (25). These modes will grow exponentially, as we shall now show, as will also $A_{t}$ and $A_{z}$, until they reach an equilibrium configuration where the quadratic terms become large enough to stop the instability.

To examine the $W$ instability, we note first that Eq. (25) is not well adapted since it was written for the usual components of $W^{\mu}$. Instead, we write an equivalent linearized action [obtained by retaining only the lowest order tems in the Lagrangian (11)] for the field $W=W_{3}^{+}$alone, namely

$$
\begin{aligned}
S=\pi \int r d r d t & \left\{\frac{\beta_{t}^{2}-\beta_{z}^{2}}{\beta_{z}^{2}+\beta_{t}^{2}}\left|W^{\prime}\right|^{2}-\frac{2 \beta_{z}^{2}+\beta_{t}^{2}}{\beta_{z}^{2}+\beta_{t}^{2}}|\dot{W}|^{2}+\frac{i \beta_{t}}{\sqrt{\beta_{z}^{2}+\beta_{t}^{2}}}\left(W^{\prime} \dot{W}^{\star}-\dot{W} W^{\prime \star}\right)\right. \\
& -g\left(s A_{z}+c Z_{z}\right)\left[\frac{\beta_{z}}{\sqrt{\beta_{z}^{2}+\beta_{t}^{2}}}\left(W^{\prime \star} W+W^{\star} W^{\prime}\right)+\frac{i \beta_{z} \beta_{t}}{\beta_{z}^{2}+\beta_{t}^{2}}\left(\dot{W} W^{\star}-\dot{W}^{\star} W\right)\right] \\
& +g\left(s A_{t}+c Z_{t}\right)\left[\frac{\beta_{t}}{\sqrt{\beta_{z}^{2}+\beta_{t}^{2}}}\left(W^{\prime \star} W+W^{\star} W^{\prime}\right)+i \frac{2 \beta_{z}^{2}+\beta_{t}^{2}}{\beta_{z}^{2}+\beta_{t}^{2}}\left(\dot{W} W^{\star}-\dot{W}^{\star} W\right)\right] \\
& \left.+\frac{2 g}{\sqrt{\beta_{z}^{2}+\beta_{t}^{2}}}\left[\beta_{z}\left(s A_{z}^{\prime}+c Z_{z}^{\prime}\right)-\beta_{t}\left(s A_{t}^{\prime}+c Z_{t}^{\prime}\right)\right]|W|^{2}\right\}
\end{aligned}
$$

whose variations yield the following Euler-Lagrange equation:

$$
a\left(W^{\prime \prime}+\frac{1}{r} W^{\prime}\right)+\left(\delta^{\prime}+\frac{1}{r} \delta\right) W-i \frac{c}{r} \dot{W}+b \ddot{W}-i \dot{\epsilon} W-2 i \epsilon \dot{W}=\sigma W,
$$

where we have set $a=\left(\beta_{t}^{2}-\beta_{z}^{2}\right) /\left(\beta_{z}^{2}+\beta_{t}^{2}\right), b=-\left(2 \beta_{z}^{2}+\beta_{t}^{2}\right) /\left(\beta_{z}^{2}+\beta_{t}^{2}\right), c=\beta_{t} / \sqrt{\beta_{z}^{2}+\beta_{t}^{2}}$

$$
\begin{gathered}
\delta(r, t)=-\frac{g}{\sqrt{\beta_{z}^{2}+\beta_{t}^{2}}}\left[\beta_{z}\left(s A_{z}+c Z_{z}\right)-\beta_{t}\left(s A_{t}+c Z_{t}\right)\right], \\
\epsilon(r, t)=\frac{g \beta_{z} \beta_{t}}{\beta_{z}^{2}+\beta_{t}^{2}}\left(s A_{z}+c Z_{z}\right)-g \frac{2 \beta_{z}+\beta_{t}}{\beta_{z}^{2}+\beta_{t}^{2}}\left(s A_{t}+Z_{t}\right),
\end{gathered}
$$

and

$$
\sigma(r)=\frac{2 g}{\sqrt{\beta_{z}^{2}+\beta_{t}^{2}}}\left[\beta_{z}\left(s A_{z}^{\prime}+c Z_{z}^{\prime}\right)+\beta_{t}\left(s A_{t}^{\prime}+c Z_{t}^{\prime}\right)\right] .
$$

Although it is impossible to solve Eq. (36), some information regarding the transition from the nonconducting state to the current-carrying state can be obtained from it if one makes an "adiabatic" hypothesis, namely assumes that the transition is slow enough that the time derivative in the gauge fields can be neglected compared to their spatial gradients. In this hypothesis, setting $W=\xi(t) \rho(r)$ into Eq. (36) yields 


$$
a \frac{\rho^{\prime \prime}+\rho^{\prime} / r}{\rho}+\delta^{\prime}+\frac{1}{r} \delta-\sigma=i \frac{\dot{\xi}}{\xi}\left(\frac{c}{r}+2 \epsilon\right)-b \frac{\ddot{\xi}}{\xi} \equiv-\zeta(r),
$$

where the function $\zeta$ depends on the radial coordinate $r$ only. Thus, the oscillatory modes $\xi \propto e^{i \omega t}$ satisfy a dispersion relation

$$
\omega^{2}-\frac{1}{b}\left(\frac{c}{r}+2 \epsilon\right) \omega+\frac{\zeta}{b}=0
$$

whose solutions have an imaginary part for $\zeta>\frac{1}{4 b}(c / r+2 \epsilon)^{2}$, i.e., in the low frequency limit (thereby justifying the "adiabatic" hypothesis). In the limit of zero frequency $(\omega \rightarrow 0)$, we can estimate roughly, in order of magnitude, the expected value of the timescale necessary for the string to become current-carrying (namely $\tau \sim \omega^{-1}$ ): assuming the current-carrier field to have an amplitude [17] $|W| \sim M_{W}$, and taking $r_{0}$ to be the typical distance over which the fields vary, then Eqs. (33) and (34) give $A_{z} \sim g s r_{0} M_{W}^{2}$, and we find

$$
\tau \sim\left(e M_{W}\right)^{-1}
$$

i.e., a time independent of the string thickness, an expected result since the background string and the electroweak fields are decoupled in the core (again, neglecting backreaction). It should be mentionned that for coupling values $f>f_{\text {crit }}$, the same mechanism actually applies, but that in this case, the initial configuration and only metastable and although the current will definitely be spontaneously generated, it will be through tunnelling. As a result, the life time of the noncurrent-carrying configuration is in fact increased by a factor depending on the strings thickness and the value of $h$ at $r=0$, these giving the order of magnitude of the expected potential barrier and its width: the standard WKB approximation then gives an extra exponential factor in Eq. (42).

\section{INTERNAL STRING STRUCTURE.}

The spontaneous current generation mechanism we have just discussed has in fact many interesting consequences, including, we believe, cosmological (notably in the framework of the vorton problem [12] which becomes even more unavoidable in this context), and in this section, we wish to emphasize a particular effect, namely that generating a current this way breaks the Lorentz boost invariance along the string spontaneously. The basic reason that this occurs is that the particle that gets trapped in the string is in fact a $W_{\mu}^{ \pm}$, i.e., a vectorial particle, and a nonvanishing VEV for a vector picks a privileged direction in space time. Also we shall exhibit the internal microscopic structure of the string and compare it with what is obtained in the simple Witten [10,17] bosonic toy model.

As was already said, the current generation phenomenon will spontaneously break the Lorentz boost symmetry along the string: before the $W$ condenses in the strings core, the energy per unit length and the tension are both equal so a boost in the $z$ direction does not change the physics of the system. Now, when a perturbation in $W$ is applied, as we have just seen, the $W$ and $A$ fields VEV increase exponentially, so the degeneracy of the stress energy tensor is raised exponentially as times passes by, until again the system reaches a stationnary configuration. It is therefore an actual spontaneous mechanism because it does not require any external field and it needs not even exist in the expanding Universe (as is the case for the usual symmetry breaking Higgs mechanism).

A stationnary configuration obtained this way consists in a $W$ field together with, as argued before, any value for the ratio $\beta_{z} / \beta_{t}$. In fact, it turns out that the only thing to know to determine (nearly) entirely the configuration is whether this ratio is less or greater than unity, for once this is known, it suffices to apply a boost along the string to remove one of the field $A_{z}$ or $A_{t}$. As a result, the only interesting cases are the magnetic case for which one can always set $\beta_{t}=0$ and $A_{t}=Z_{t}=0$, the electric case having $\beta_{z}=0$ and $A_{z}=Z_{z}=0$, and the null or lightlike case with $\beta_{z}=\beta_{t} \equiv \beta, A_{z}=A_{t} \equiv A$ and $Z_{z}=Z_{t} \equiv Z$, as can be seen on Eqs. (33) and (34). It can then be shown [17.22] that the most general configuration will be

$$
W=\Upsilon(r) e^{i \psi(z, t)},
$$

where, without lack of generality [17], the phase function $\psi$ could be chosen as $\psi=\omega t-k z$. The "energy per unit length" is then

$$
\begin{aligned}
\tilde{U}=2 \pi \int r d r & \left\{\frac{1}{2} h^{\prime 2}+\varphi^{\prime 2}+\frac{\varphi^{2} Q^{2}}{r^{2}}+\frac{2 Q^{\prime 2}}{r^{2} q^{2}}+\frac{\lambda_{H}}{4}\left(h^{2}-v_{h}^{2}\right)^{2}+\lambda_{\phi}\left(\varphi^{2}-\frac{v_{\phi}^{2}}{2}\right)^{2}+\frac{1}{2} f\left(h^{2}-v_{h}^{2}\right)\left(\varphi^{2}-\frac{v_{\phi}^{2}}{2}\right)+\frac{1}{2} \Upsilon^{\prime 2}\right. \\
& +\frac{1}{2}\left[\left(\partial_{z} \psi\right)^{2}+\left(\partial_{t} \psi\right)^{2}+\frac{\left(\beta_{t} \partial_{z} \psi+\beta_{z} \partial_{t} \psi\right)^{2}}{\beta_{z}^{2}+\beta_{t}^{2}}\right] \Upsilon^{2}+\frac{\beta_{z} \partial_{z} \psi+\beta_{t} \partial_{t} \psi}{\sqrt{\beta_{z}^{2}+\beta_{t}^{2}}} \Upsilon \Upsilon^{\prime}+\frac{1}{2}\left(A_{z}^{\prime 2}+A_{t}^{\prime 2}+Z_{z}^{\prime 2}+Z_{t}^{\prime 2}\right)
\end{aligned}
$$




$$
\begin{aligned}
& +\frac{g^{2}}{8 c^{2}} h^{2}\left(Z_{z}^{2}+Z_{t}^{2}\right)+\frac{1}{4} g^{2} h^{2} \Upsilon^{2}+\frac{1}{2} g^{2} \Upsilon^{4}+g^{2} \Upsilon^{2}\left[\left(s A_{z}+c Z_{z}\right)^{2}+\left(s A_{t}+c Z_{t}\right)^{2}\right] \\
& +g\left(s A_{z}+c Z_{z}\right)\left\{\frac{\beta_{z} \Upsilon \Upsilon^{\prime}}{\sqrt{\beta_{z}^{2}+\beta_{t}^{2}}}+\left[\partial_{z} \psi+\frac{\beta_{t}}{\beta_{z}^{2}+\beta_{t}^{2}}\left(\beta_{t} \partial_{z} \psi+\beta_{z} \partial_{t} \psi\right)\right] \Upsilon^{2}\right\} \\
& +g\left(s A_{t}+c Z_{t}\right)\left\{\frac{\beta_{t} \Upsilon \Upsilon^{\prime}}{\sqrt{\beta_{z}^{2}+\beta_{t}^{2}}}+\left[\partial_{t} \psi-\frac{\beta_{z}}{\beta_{z}^{2}+\beta_{t}^{2}}\left(\beta_{t} \partial_{z} \psi+\beta_{z} \partial_{t} \psi\right)\right] \Upsilon^{2}\right\} \\
& \left.-\frac{g^{2} \Upsilon^{2}}{2\left(\beta_{z}^{2}+\beta_{t}^{2}\right)}\left[\left(s A_{z}+c Z_{z}\right) \beta_{z}+\left(s A_{t}+c Z_{t}\right) \beta_{t}\right]^{2}-\frac{g \Upsilon^{2}}{\sqrt{\beta_{z}^{2}+\beta_{t}^{2}}}\left[\left(s A_{z}^{\prime}+c Z_{z}^{\prime}\right) \beta_{z}+\left(s A_{t}^{\prime}+c Z_{t}^{\prime}\right) \beta_{t}\right]\right\}
\end{aligned}
$$

and the "tension"

$$
\begin{aligned}
\tilde{T}=2 \pi \int r d r & \left\{\frac{1}{2} h^{\prime 2}+\varphi^{\prime 2}+\frac{\varphi^{2} Q^{2}}{r^{2}}+\frac{2 Q^{\prime 2}}{r^{2} q^{2}}+\frac{\lambda_{H}}{4}\left(h^{2}-v_{h}^{2}\right)^{2}+\lambda_{\phi}\left(\varphi^{2}-\frac{v_{\phi}^{2}}{2}\right)^{2}+\frac{1}{2} f\left(h^{2}-v_{h}^{2}\right)\left(\varphi^{2}-\frac{v_{\phi}^{2}}{2}\right)-\frac{1}{2} \Upsilon^{\prime 2}\right. \\
& -\frac{1}{2}\left[\left(\partial_{z} \psi\right)^{2}+\left(\partial_{t} \psi\right)^{2}+\frac{\left(\beta_{t} \partial_{z} \psi+\beta_{z} \partial_{t} \psi\right)^{2}}{\beta_{z}^{2}+\beta_{t}^{2}}\right] \Upsilon^{2}-\frac{\beta_{z} \partial_{z} \psi+\beta_{t} \partial_{t} \psi}{\sqrt{\beta_{z}^{2}+\beta_{t}^{2}}} \Upsilon \Upsilon^{\prime}-\frac{1}{2}\left(A_{z}^{\prime 2}+A_{t}^{\prime 2}+Z_{z}^{\prime 2}+Z_{t}^{\prime 2}\right) \\
& -\frac{g^{2}}{8 c^{2}} h^{2}\left(Z_{z}^{2}+Z_{t}^{2}\right)-\frac{1}{2} g^{2} \Upsilon^{4}-g\left(s A_{z}+c Z_{z}\right)\left\{\frac{\beta_{z} \Upsilon \Upsilon^{\prime}}{\sqrt{\beta_{z}^{2}+\beta_{t}^{2}}}+\left[\partial_{z} \psi-\frac{\beta_{t}}{\beta_{z}^{2}+\beta_{t}^{2}}\left(\beta_{t} \partial_{z} \psi+\beta_{z} \partial_{t} \psi\right)\right] \Upsilon^{2}\right\} \\
& -g\left(s A_{t}+c Z_{t}\right)\left\{\frac{\beta_{t} \Upsilon \Upsilon^{\prime}}{\sqrt{\beta_{z}^{2}+\beta_{t}^{2}}}+\left[\partial_{t} \psi+\frac{\beta_{z}}{\beta_{z}^{2}+\beta_{t}^{2}}\left(\beta_{t} \partial_{z} \psi+\beta_{z} \partial_{t} \psi\right)\right] \Upsilon^{2}\right\} \\
& \left.-\frac{g^{2} \Upsilon^{2}}{2\left(\beta_{z}^{2}+\beta_{t}^{2}\right)}\left[\left(s A_{z}+c Z_{z}\right) \beta_{z}-\left(s A_{t}+c Z_{t}\right) \beta_{t}\right]^{2}+\frac{g \Upsilon^{2}}{\sqrt{\beta_{z}^{2}+\beta_{t}^{2}}}\left[\left(s A_{z}^{\prime}+c Z_{z}^{\prime}\right) \beta_{z}+\left(s A_{t}^{\prime}+c Z_{t}^{\prime}\right) \beta_{t}\right]\right\},
\end{aligned}
$$

where the quotes [and the subsequent tildes in Eqs. (45) and (47)] in the previous denominations for the energy per unit length and tension come from the fact that the stress tensor also has a nondiagonal part

$$
\begin{aligned}
A \equiv & \int d^{2} x T^{z t}=2 \pi \int r d r\left\{-\frac{g^{2}}{4 c^{2}} h^{2} Z_{t} Z_{z}-\frac{g^{2}}{4} h^{2}\left(W_{t}^{+} W_{z}^{-}+W_{z}^{+} W_{t}^{-}\right)-A_{t}^{\prime} A_{z}^{\prime}-Z_{t}^{\prime} Z_{z}^{\prime}-W_{r t}^{+} W_{r z}^{-}-W_{r z}^{+} W_{r t}^{-}\right. \\
& -i g\left[\left(s A_{z}+c Z_{z}\right)\left(W_{r t}^{-} W_{r}^{+}-W_{r t}^{+} W_{r}^{-}\right)+\left(s A_{t}+c Z_{t}\right)\left(W_{r z}^{-} W_{r}^{+}-W_{r z}^{+} W_{r}^{-}\right)\right. \\
& \left.\quad+\left(s A_{z}^{\prime}+c Z_{z}^{\prime}\right)\left(W_{r}^{-} W_{t}^{+}-W_{r}^{+} W_{t}^{-}\right)+\left(s A_{t}^{\prime}+c Z_{t}^{\prime}\right)\left(W_{r}^{-} W_{z}^{+}-W_{r}^{+} W_{z}^{-}\right)\right] \\
& \left.+g^{2}\left[\left(W_{r}^{+}\right)^{2} W_{t}^{-} W_{z}^{-}+\left(W_{r}^{-}\right)^{2} W_{t}^{+} W_{z}^{+}-\left|W_{r}\right|^{2}\left(W_{z}^{+} W_{t}^{-}+W_{t}^{+} W_{z}^{-}\right)-2\left|W_{r}\right|^{2}\left(s A_{z}+c Z_{z}\right)\left(s A_{t}+c Z_{t}\right)\right]\right\},
\end{aligned}
$$

and this part can be removed by choosing the reference frame in which either $\beta_{t}$ or $\beta_{z}$ vanishes, which is always possible provided they are not equal, i.e., provided the current is not lightlike. In this case however, the eigenvectors $u^{\mu}$ and $v^{\mu}$ of the stress energy tensor (10) are both null and the definition of the eigenvalues as energy per unit length and tension becomes unclear. Investigation of such lightlike currents in strings is posponed for future work [37].

Therefore, in general, as was already discussed in Ref. [22], the knowledge of the energy per unit length and the tension in the particular cases $\beta_{t}=0, \beta_{z}>0$ and $\beta_{z}=0, \beta_{t}>0$ will be sufficient for macroscopic applications. For these two cases, it turns out that $U$ and $T$ (without the tilde, i.e., after diagonalization of the stress tensor, this operation corresponding to choosing $\left[\beta_{z}=0, \partial_{z} \psi=0\right]$ or $\left.\left[\beta_{t}=0, \partial_{t} \psi=0\right]\right)$ actually depend on the gauge fields $A_{\mu}$ and $Z_{\mu}$ only through the new field functions

$$
\nu P(r)=\partial_{a} \psi+g\left(s A_{a}+c Z_{a}\right),
$$

and the orthogonal field

$$
\nu^{\prime} R(r)=-\frac{c}{s} \partial_{a} \psi+g\left(s Z_{a}-c A_{a}\right),
$$

where $a$ denotes either $z$ or $t$ depending on to whether one considers a magnetic or an electric state respectively, and where the parameter $\nu^{\prime}$ is not in fact arbitrary since it is given by $\nu^{\prime}=-c \nu / s$. As a result, the realistic string model given by the Lagrangian (1) is nearly as simple as the Witten [10,17 bosonic toy model in the sense that out 
of the initial 18 field functions $\left(\varphi, h, C_{\mu}, W_{\mu}^{ \pm}, A_{\mu}\right.$, and $R_{\mu}$ ), the internal microscopic structure is fully determined by the knowledge of 6 field functions $[\varphi(r), h(r), Q(r), \Upsilon(r), P(r)$ and $R(r)]$ and two free parameters, namely the winding number $n$ and the state parameter $\nu$. This is to be compared to the original Witten bosonic model whose structure needs the knowledge of already 4 field functions and the same two free parameters, and with very similar field equations. Thus we believe that, apart from the spontaneous current generation mechanism discussed in the present article, most qualitative conclusions regarding this simple model should apply as well to this more realistic model.

\section{CONCLUSIONS.}

In examining the internal structure of cosmic strings arising in the most simple string-forming extension of the standard electroweak model, we have found that, because of the nonabelian nature of $S U(2) \times U(1)$, the field $W$ can condense spontaneously in a strings core if the coupling constant between the string-forming Higgs field and the usual $S U(2)$ doublet Higgs is less than a critical value. This phenomenon can be understood in the following way: for certain values of the coupling constants between the string forming Higgs field $\Phi$ and the $S U(2)$ doublet Higgs field $H$, the latter has a vanishing VEV close to the strings core, so the initial $S U(2) \times U(1)$ symmetry is restored. Therefore, the intermediate vector bosons, just like the photon, remains massless in this region. Any exitation of the photon field will thus have enough energy to generate a pair $W^{+} W^{-}$through vacuum fluctuations. In turn, the effectively massless $W$ particle, being charged, is responsible for the existence of a nonvanishing electromagnetic field. This turns out to be in fact an unstable fluctuation mode, and nonzero VEV for $W$ and the photon therefore build up spontaneously.

By using the field equations for the electroweak fields in the symmetry restored region, we have been able to exhibit explicitely this instability, and to estimate what we believe to be a lower bound on the time necessary for the current to be generated. This timescale is, as expected, independent of the underlying string parameters provided the latter are such that the symmetry restoration mechanism actually occurs. It should be remarked that because the phenomenon here described is essentially electromagnetic and involves only the $W$ particle, the timescale found could have been deduced on dimensionnal ground, namely $\tau \sim\left(e M_{W}\right)^{-1}$. Although this is a huge time compared to the characteristic length of the string if the underlying string forming theory is at GUT scale, it is still sufficiently short to be irrelevant for cosmological considerations. Thus, current-carrying strings seem quite generic in string-forming GUT models since the potential (2) is in fact very general even as a low energy limit and, as we have seen, the current formation mechanism is independent of the background string structure.

Considering the results of Ref. [22] and the present calculation, it can be concluded that for any value value of the coupling between the string forming theory and the electroweak fields, the resulting strings are superconducting in the sense of Witten, whether the current builds up through tunneling (high frequencies metastability [22]) or instability. Thus, if cosmic string exist, and if they are not arbitrarily decoupled from the low energy physics (a requirement of "naturalness"), then, they are superconducting. Since the present knowledge in high energy physics tells us that approximately half of the plausible GUT theories contains cosmic strings, it means that we can estimate the existence probability of superconducting cosmic string to be also of the order $1 / 2$.

A final remark seems appropriate at this point: the current generation we have exhibited here relies in fact entirely on the nonabelian nature of $S U(2)$. This means that for a string forming GUT, it will exist as well since GUT models usually involve large unifying groups with nonabelian couplings, and various Higgses. Therefore, gauge bosons having masses of the order of the GUT scale should spontaneously condense in the string core, within a timescale given this time by $\tau \sim\left(g M_{G U T}\right)^{-1}$, where $g$ is the GUT group coupling constant. The cosmological relevance of such effects is then obvious since many of these gauge bosons are responsible for baryon number violation, so that in particular, these strings would enhance the primordial baryon number asymmetry.

\section{ACKNOWLEDGEMENTS}

I wish to thank B. Carter, A.-C. Davis, P. Fayet, J. Garriga, M. Hindmarsh, E. P. S. Shellard and A. Vilenkin for many interesting and improving discussions. This work was supported by SERC grant \# 15091-AOZ-L9. 
[1] T. W. Kibble, J. Phys. A 9, 1387 (1976); Phys. Rep. 67, 183 (1980)

[2] M.Hindmarsh, A.-C. Davis, R. H. Brandenberger, preprint DAMTP-R93/13, BROWN-HET-902; P. Peter, preprint DAMTP-R93/31

[3] A. Vilenkin, in Proceeding of the 14th Texas Symposium on Relativistic Astrophysics, preprint TUTP-89-1

[4] A. Vilenkin, Phys. Rep. 121, 263 (1985)

[5] R. Davis, Phys. Rev. D 35, 3705 (1987); N. Turok, Phys. Rev. Lett. 63, 2225 (1989); D. Spergel, N. turok, W. Press, B. Ryden, Phys. Rev. D 43, 1038 (1991); J. Borrill, E. Copeland, A. Liddle, Phys. Lett. B 258, 310 (1991); L. Perivolaropoulos, Phys. Rev. D 46, 1858 (1992)

[6] T. Hara, P. Mähönen, S. Miyoshi, Astrophys. J. 414, 421 (1993) and references therein; D. Coulson, P. Ferreira, P. Graham, N. Turok, preprint HUP-TH-93/1393

[7] D. Bennet, F. R. Bouchet, Phys. Rev. Lett. 60, 257 (1988); Phys. Rev. D 41, 2408 (1990); A. Albrecht, N. Turok, ibid 40, 973 (1989); B. Allen, E. P. S. Shellard, ibid 64, 119 (1990); A. Stebbins, S. Veeraraghavan, R. Brandenberger, J. Silk, N. Turok, Astrophys. J. 322, 1 (1987); R. H. Brandenberger, preprint BROWN-HET-906

[8] J. F. Smoot et al., Astrophys. J. 396, L1 (1992)

[9] M. Hindmarsh, A. Wray, Phys. Lett. B 251, 498 (1990); P. Peter, Class. Quantum Grav. (1993)

[10] E. Witten, Nucl. Phys. B 249, 557 (1985)

[11] J. P. Ostriker, C. Thompson, E. Witten, Phys. Lett. B 180, 231 (1986)

[12] R. L. Davis, E. P. S. Shellard, Phys. Rev. D 38, 4722 (1988); Nucl. Phys. B 323, 209 (1989)

[13] B. Carter, Phys. Lett. B 238, 166 (1990)

[14] B. Carter, in The early Universe and Cosmic Structures, Proceedings of the Xth Moriond Astrophysics Meeting, Les Arcs, France, 1990, Edited by A. Blanchard and J. Tran Thanh Vân (Editions Frontières, Gif-sur-Yvette, 1990)

[15] B. Carter, X. Martin, Ann. Phys. 227, 151 (1993)

[16] P. Peter, Phys. Lett. B 298, 60 (1993)

[17] E. Copeland, M. Hindmarsh, N. Turok, Phys. Rev. Lett. 58, 1910 (1987); R. L. Davis, E. P. S. Shellard, Phys. Lett. B 209, 485 (1988); ibid 207, 404 (1988); C. T. Hill, H. M. Hodges, M. S. Turner, Phys. Rev. D 37, 263 (1988); A. Babul, T. Piran, D. N. Spergel, Phys. Lett. B 202, 307 (1988); E. Copeland, D. Haws, M. Hindmarsh, N. Turok, Nucl. Phys. B 306, 908 (1988); D. Haws, M. Hindmarsh, N. Turok, Phys. Lett. B 209, 255 (1988) P. Peter, Phys. Rev. D 45, 1091 (1992); ibid 46, 3335 (1992)

[18] P. Peter, Phys. Rev. D 47, 3169 (1993)

[19] B. Carter, in Formation and Evolution of Cosmic Strings, Edited by G. W. Gibbons, S. W. Hawking and T. Vachaspati (Cambridge University Press, Cambridge, England, 1990); Phys. Lett. B 228, 466 (1989)

[20] T. M. Helliwell, D. A. Konkowski, Phys. Rev. D 34, 1918 (1986); I. Moss, S. Poletti, Phys. Lett. B 199, 34 (1987); A. Babul, T. Piran, D. N. Spergel, ibid 209, 477 (1988); P. Amsterdamski, P. Laguna-Castillo, Phys. Rev. D 37, 877 (1988); B. Linet, Class. Quantum Grav. 6, 435 (1989); P. Peter, D. Puy, Phys. Rev. D 48, (1993)

[21] M. M. Salomaa, G. E. Volovik, Rev. Mod. Phys. 59, 533 (1987)

[22] P. Peter, Phys. Rev. D 46, 3322 (1992) and references therein; W. B. Perkins, A.-C. Davis, Nucl. Phys. B 406, 377 (1993)

[23] S.L. Glashow, Nucl. Phys. 22, 579 (1961); A. Salam, J.C. Ward, Phys. Lett. 13, 168 (1964); S. Weinberg, Phys. Rev. Lett. 19, 1264 (1967)

[24] T.W.B. Kibble, G. Lazarides, Q. Shafi, Phys. Lett. B 113, 237 (1982); P. Fayet, S. Ferrara, Phys. Rep. 32, 249 (1977); P. Fayet, Nucl. Phys. B 347, 743 (1990) R. W. Robinett, J. Rosner, Phys. Rev. D 25, 3036 (1982); C. N. Leung, J. Rosner, Phys. Rev. D 29, 2132 (1984) F. Del Aguila, M. Quirós, F. Zwirner, Nucl. Phys. B 287, 419 (1987); T. M. Bibilashvili, G. R. Dvali, Phys. Lett. B 263, 359 (1991); ibid 248, 259 (1990)

[25] L. S. Durkin, P. Langacker, Phys. Lett. B 166, 436 (1986); Particle Data Group, Phys. Rev. D 45, Part 2 (1992); Phys. Lett. B 276, 247 (1992); K. Hagiwara, R. Najima, M. Sakuda, N. Terunuma, Phys. Rev. D 41, 815 (1990); M. C. GonzalezGarcia, J. F. W. Valle, Phys. Rev. B 236, 360 (1990); U. Amaldi et al.,Phys. Rev. D 36, 1385 (1987); F. Del Aguila, J. M. Moreno, M. Quirós, Phys. Lett. B 254, 497 (1991); G. Altarelli et al., Phys. Lett. B 263, 459 (1991); P. Langacker, M. Luo, Phys. Rev. D 45, 278 (1992)

[26] H. B. Nielsen, P. Olesen, Nucl. Phys. B 61, 45 (1973)

[27] M. James, L. Perivolaropoulos, T. Vachaspati, Phys. Rev. D 46, R5232 (1992)

[28] Y. Nambu, Nucl. Phys. B 130, 504 (1977); M. B. Einhorn, R. Savit, Phys. Lett. B 77, 295 (1978); V. Soni, Phys. Lett. B 93, 101 (1980); T. Vachaspati, Phys. Rev. Lett. 68, 1977 (1992)

[29] J. Gasser, H. Leutwyler, Nucl. Phys. B 250, 465 (1985)

[30] R. Holtman, S. Hsu, T. Vachaspati, R. Watkins, Phys. Rev. D 46, 5352 (1992)

[31] L. Dolan, R. Jackiw, Phys. Rev. D 9, 3320 (1974); S. Weinberg, Phys. Rev. D 9, 3357 (1974); D. Bailin, A. Love, Introduction to gauge field theory, Adam Hilger Ed. (1986)

[32] M. Reuter, C. Wetterich, Nucl. Phys. B 408, 91 (1993)

[33] L. Jacobs, C. Rebbi, Phys. Rev. B 19, 4486 (1979)

[34] S. L. Adler, T. Piran, Rev. Mod. Phys. 56, 1 (1984)

[35] P. Laguna, R. Matzner, Phys. Rev. D 41, 1751 (1990)

[36] J. Ambjørn, P. Olesen, Nucl. Phys. B 315, 606 (1989); Phys. Lett. B 218, 67 (1989) and references therein 
[37] J. Garriga, P. Peter, in preparation

FIG. 1. Field configuration around the vortex exhibiting the $\mathrm{SU}(2) \times \mathrm{U}(1)$ restoration for $\varphi<\varphi_{\min }$. This picture was drawn using a large value for $|f|$ in order to have a large symmetry restoration region. 
Higgs fields configuration around the vortex

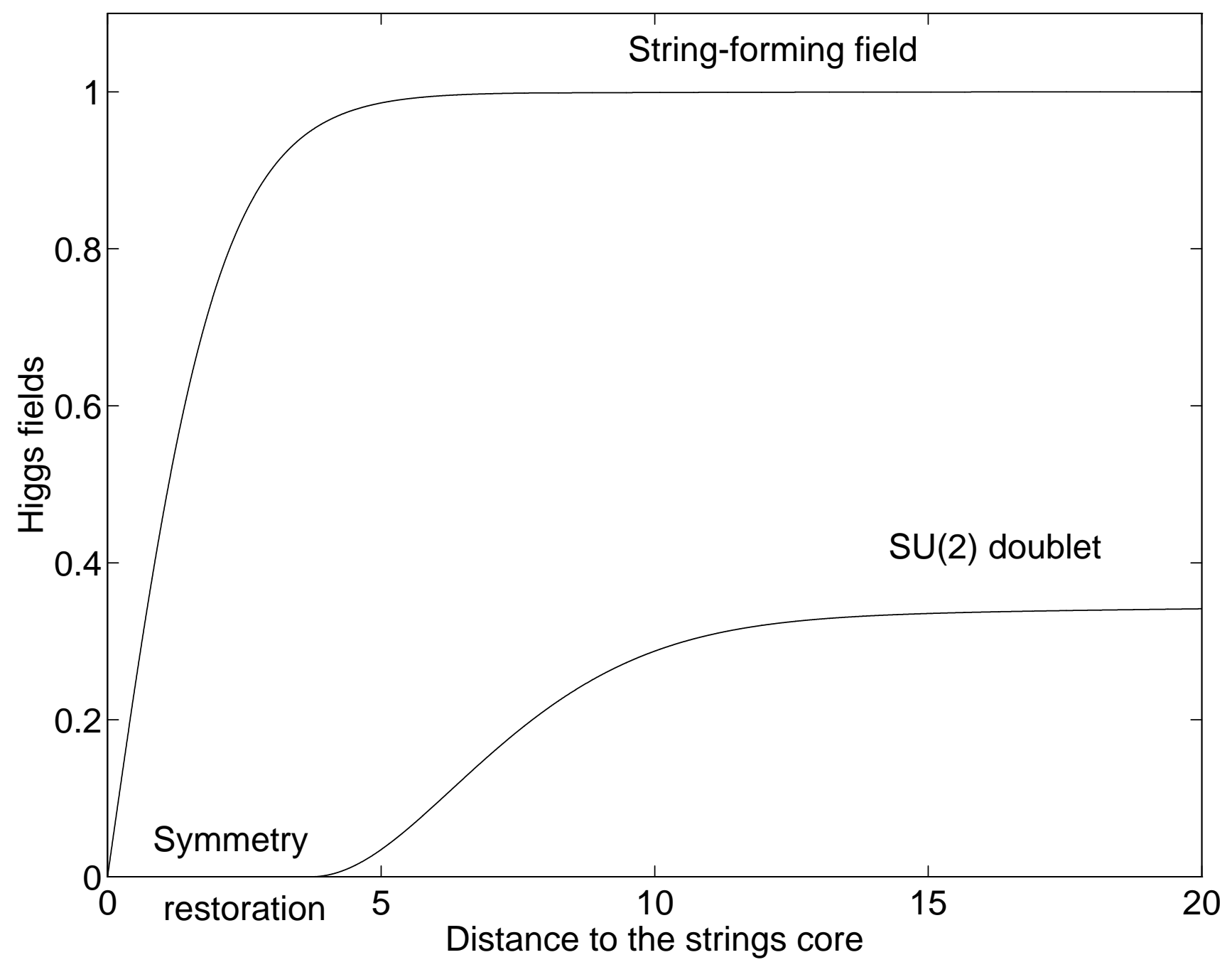


This figure "fig1-1.png" is available in "png" format from: http://arxiv.org/ps/hep-ph/9312280v1 